\title{
Helicobacter pylori ve inflamatuvar bağırsak hastalığı arasındaki ilişki
}

\author{
Relationship between Helicobacter pylori infection and inflammatory bowel disease
}

\author{
(DEmre GERÇEKER ${ }^{1}$, (D) Serkan CERRAH ${ }^{2}$, (D)Ahmed Ramiz BAYKAN² \\ İzmir Özel Gazi Hastanesi ${ }^{1}$ Gastroenteroloji Bölümü, İzmir \\ Erzurum Eğitim ve Araştırma Hastanesi ${ }^{2}$ Gastroenteroloji Kliniği, Erzurum
}

Giriş ve Amaç: Kronik Helicobacter pylori enfeksiyonunun, sistematik immün toleransı indükleyerek ve inflamatuvar tepkileri baskılayarak inflamatuvar bağırsak hastalığına karşı korumada bir rol oynadığı ileri sürülmektedir. Çalışmanın birincil amacı inflamatuvar bağırsak hastalığı olgularında Helicobacter pylori sıklığını saptamaktır. ikincil amacı ise inflamatuvar bağırsak hastalığı ve Helicobacter pylori enfeksiyonu arasındaki ilişkiyi araştırmaktır. Gereç ve Yöntem: Inflamatuvar bağırsak hastalığı tanısı ile takip edilen, dispepsi nedeni ile üst gastrointestinal sistem endoskopisi yapılan 18 yaş üzeri hastaların verileri retrospektif olarak incelenerek olgular çalışmaya dahil edildi. Kontrol grubu inflamatuvar bağırsak hastalığı tanısı olmayan ve dispepsi nedeni ile üst gastrointestinal sistem endoskopisi yapılan olgulardan oluşturuldu. Tüm hastaların yaş, cinsiyet, üst gastrointestinal sistem endoskopide alınan biyopsi örneklerinin patolojik değerlendirme sonucundaki Helicobacter pylori varlığı, atrofik gastrit ve intestinal metaplazi varlığı bulguları kaydedildi. Her iki grup Helicobacter pylori sıklığı ve histopatolojik bulgular (intestinal metaplazi ve atrofik gastrit varlığı) yönünden karşılaştıılldı. Bulgular: Yüz altmış inflamatuvar bağırsak hastası ve 60 kontrol olmak üzere toplam 220 olgu çalısmaya dahil edildi. Inflamatuvar bağırsak hastalığı olgularının \%53.8'i ( $n=86)$ ülseratif kolit, \%46.2'si ( $n=74)$ Crohn hastalığı tanıı olgulardı. Inflamatuvar bağırsak hastalı̆ı grubunda Helicobacter pylori oranı kontrol grubu ile karşılaştıııldığında anlamlı olarak daha düşük saptandı (\%52.5'e karşı \%73.3 ve $p=0.005)$. Inflamatuvar bağırsak hastalığı grubunda atrofik gastrit oranı kontrol grubu ile karşılaştıııldığında anlamlı olarak daha düşük bulundu (\%3.1'e karşı $\% 15$ ve $p=0.001$ ). Inflamatuvar bağırsak hastalı̆ı grubunda intestinal metaplazi oranı kontrol grubu ile karşılaştıııldığında anlamlı olarak daha düşüktü (\%1.9'a karşı \%11.7 ve p =0.002). Sonuç: Inflamatuvar bağırsak hastalığı grubunda anlamlı olarak daha düşük oranda Helicobacter pylori pozitifliği saptanmıştır. Aynı şekilde, inflamatuvar bağırsak hastalığı grubunda daha düşük oranda atrofik gastrit ve intestinal metaplazi varlığı saptanmıştır. Inflamatuvar bağırsak hastalığı olgularında tedavide kullanılan antibiyotiklerin Helicobacter pylori sıklığını azaltarak daha az sıklıkta atrofik gastrit ve intestinal metaplazi gelişimine yol açtığını düşünmekteyiz.

Anahtar kelimeler: Crohn hastalı̆ı, Helicobacter pylori, inflamatuvar bağırsak hastalı̆̆ı ülseratif kolit

iletişim: Emre GERÇEKER

İmir Özel Gazi Hastanesi Gastroenteroloji Bölümü, Alsancak,

Kahramanlar Mh., Nevzat Güzelırmak Sk. No:29, 35230 Konak, Izmir

Tel: +90 23246479 79/2037 • Faks: +90 2324648369 - E-mail: dr.emre.gerceker@gmail.com
Background and Aims: It has been suggested that chronic Helicobacter pylori infection plays a protective role against inflammatory bowel disease by inducing systemic immune tolerance and suppressing inflammatory responses. The primary aim of the study was to determine the frequency of Helicobacter pylori in inflammatory bowel disease patients. A secondary aim was to investigate the relationship between inflammatory bowel disease and Helicobacter pylori infection. Materials and Methods: Patients over 18 years of age who were in follow-up with a diagnosis of inflammatory bowel disease and who underwent upper gastrointestinal system endoscopy for dyspepsia were included in the study and their data retrospectively analyzed. The control group consisted of patients who were not diagnosed with inflammatory bowel disease and had upper gastrointestinal system endoscopy for dyspepsia. For all patients, age; gender; and the presence of Helicobacter pylori, atrophic gastritis, and intestinal metaplasia as a result of pathological evaluation of biopsy samples taken by upper gastrointestinal system endoscopy were recorded. Both groups were compared in terms of Helicobacter pylori frequency and histopathological findings (presence of intestinal metaplasia and atrophic gastritis). Results: A total of 220 cases, including 160 inflammatory bowel disease and 60 controls, were included in the study. In all, 53.8\% $(n=86)$ of inflammatory bowel disease patients were diagnosed with ulcerative colitis and $46.2 \%$ ( $n$ = 74) with Crohn's disease. The rate of Helicobacter pylori was found to be significantly lower in the inflammatory bowel disease group compared with the control group (52.5\% versus $73.3 \%$ and $p=0.005)$. The rate of atrophic gastritis was significantly lower in the inflammatory bowel disease group compared with the control group (3.1\% vs 15\% and $p=0.001$ ). The intestinal metaplasia rate was significantly lower in the inflammatory bowel disease group compared with the control group $(1.9 \%$ vs $11.7 \%$ and $p=0.002)$. Conclusion: Helicobacter pylori positivity was found at a significantly lower rate in the inflammatory bowel disease group. The presence of atrophic gastritis and intestinal metaplasia was found to be lower in the inflammatory bowel disease group. We believe that antibiotics used in the routine treatment of inflammatory bowel disease patients decrease the frequency of Helicobacter pylori and lead to less frequent development of atrophic gastritis and intestinal metaplasia.

Key words: Crohn's disease, Helicobacter pylori, inflammatory bowel disease, ulcerative colitis 


\section{Giriş,}

Helicobacter pylori (H. pylori) spiral, mikroaerofilik, gram negatif bir bakteridir. H. pylori insanlardaki en sık kronik enfeksiyon sebebidir. Mide mukozasında özgül hareketliliği, mikroaerobik metabolizması ve anti-asit aktivitesi sayesinde kolonize olur. Kolonizasyon sonrası H. pylori birçok mekanizma ile konak defansı yenerek kronik mukozal enflamasyona neden olmaktadır. Kronik mukozal H. pylori enfeksiyonu lokal immün reaksiyon ile kronik gastrit, peptik ülser, gastrik lenfoma ve gastrik kanser gibi gastroduodenal hastalıkların patogenezinde önemli rol oynamaktadır (1).

Gastrik kanser, dünya çapında kansere bağlı ölümlerin ikinci sırada gelen nedenidir (2). Gastrik kanser, çeşitli genetik ve epigenetik değişikliklerin progresif olarak artmasıyla inflamasyon varlığında mukozadan gelişir ve gastrit-atrofi-metaplazi-displazi-kanser dizisini izler $(3,4)$. Atrofik gastrit (AG), intestinal metaplazi (IM) ve H. pylori, gastrik kanser gelişimi için risk faktörleridir (5). Ayrıca, $H$. pylori enfeksiyonu, kronik AG ve IM ile güçlü bir ilişki gösterir (6).

Inflamatuvar bağırsak hastalığı (IBH), [Crohn hastalığı $(\mathrm{CH})$ ve ülseratif kolit (ÜK)] gastrointestinal sistemin kronik, nüks ve remisyonlar ile seyreden hastalığıdır. IBH patogenezi günümüzde hala tam olarak anlaşılmamışır. İBH patogenezi alanındaki çoğu çalışma, etiyolojisini immün disfonksiyon, konağın genetik duyarlıı̆̆ı ve çevresel risk faktörleri arasındaki karmaşık etkileşimlere bağlamaktadır $(7,8)$. H. pylori ve İBH arasındaki ilişki tartışmalıdır. Literatür çalışmaları incelendiğinde $H$. pylori ile IBH arasında negatif korelasyon gözlenmektedir (7). Bazı araştırmacılar, İBH'da H. pylori insidansının sağlıklı popülasyonlara göre daha düşük olduğunu bildirmişlerdir. İBH'da bu düşük H. pylori oranının olası nedenleri IBH'nın immünopatolojik özellikleri, İBH'da kullanılan 5-aminosalisilik asit (5-ASA) ve antibiyotikler gibi ilaçlardır (9-12).

Bu çalışmanın birincil amacı dispepsi vb. yakınmaları ile üst gastrointestinal sistem (GIS) endoskopi tetkiki yapıIan IBH hastalarında H. pylori sıklığını saptamaktır. İkincil amacı ise İB ve $H$. pylori enfeksiyonu arasındaki ilişkiyi araştırmaktır.

\section{GEREÇ ve YÖNTEM}

Hastanemiz gastroenteroloji bölümünde IBH tanısı ile takip edilen ve 2015-2019 yılları arasında dispepsi nedeni ile üst GiS endoskopisi yapılan 18 yaş üzeri hastaların verileri retrospektif olarak incelenerek olgular çalışmaya dahil edildi. Kontrol grubu IBH tanısı olmayan ve dispepsi nedeni ile üst GiS endoskopisi yapılan olgulardan oluştu- ruldu. Tüm hastaların yaş, cinsiyet, üst GiS endoskopide alınan biyopsi örneklerinin patolojik değerlendirme sonucundaki H. pylori varlığı, AG varlı̆ı ve İM varlığı bulguları kaydedildi. Alınan biyopsi örnekleri formalin içinde fikse edildi, ardından hematoksilen eozin ve $H$. pylori araştııılması için modifiye Giemsa ile boyandı. Tüm preparatlar patolog tarafından incelendi ve histopatolojik değerlendirme, Sydney-Houston sistemindeki parametrelere göre gerçekleștirildi. Her iki grup H. pylori sıklı̆ı̆ ve histopatolojik bulgular (IM ve AG varlığı) yönünden karşılaştırıldı. Ayrıca IBH hasta grubu ÜK ve $\mathrm{CH}$ olmak üzere alt gruplara ayrıldı. Her iki alt grup H. pylori sıklığı ve histopatolojik bulgular (IM ve AG varlığı) yönünden karşılaştırıldı.

\section{İstatistiksel Analiz}

Parametreler 'SPSS 22 for Windows' istatistik programı kullanılarak yapıldı. Kategorik (nominal) değerler yüzde (\%) olarak ifade edildi ve ki-kare testi $(\chi 2)$ ile karşılaş̧ıııdı. Sürekli sayısal (kantitatif) değerler ortalama \pm standart sapma (SD) ile ifade edildi. Kantitatif değişkenler 'Student t-testi' ve 'Anova' ile karşılaştıııldı. $p<0.05$ ise istatistiksel olarak anlamlılık olarak belirlendi.

\section{Etik Kurul}

Bu çalışma Erzurum Bölge Eğitim ve Araştırma Hastanesi Klinik ve Araştırmalar Etik Kurulunun 2019/16-148 karar no'lu onayı sonrasında gerçekleştirilmiştir.

\section{BULGULAR}

Yüz altmış IBH ve 60 kontrol olmak üzere toplam 220 olgu çalışmaya dahil edildi. IBH hastalarının \%53.8'i ( $n=$ 86) ÜK, \%46.2'si $(n=74) \mathrm{CH}$ tanılı olgulardı. Olguların $\% 52.7$ 'si kadın cinsiyete sahipti. Çalışma popülasyonunun yaş ortalaması $40.94 \pm 12.86$ saptandı. Tüm çalışma popülasyonunda $H$. pylori pozitifliği $\% 58.2$ bulundu. Tüm olgular içinde AG oranı \%6.4, iM oranı \%4.5 saptandı. Tablo 1'de tüm çalışma grubunun demografik verileri özetlenmiştir.

IBH ve kontrol grupları yaş ve cinsiyet dağııımı açısından benzerdi. İH grubunda H. pylori oranı kontrol grubu ile karşılaştııılığında anlamlı olarak daha düşük saptandı (\%52.5'e karşı \%73.3 ve $p=0.005$ ). IBH grubunda AG oranı kontrol grubu ile karşılaştırıldığında anlamlı olarak daha düşük bulundu (\%3.1'e karşı \%15 ve $p=0.001$ ). IBH grubunda IM oranı kontrol grubu ile karşılaştıııldğında anlamlı olarak daha düşük saptandı (\%1.9'a karşı $\% 11.7$ ve $p=0.002$ ).

ÜK grubunda $H$. pylori oranı kontrol grubu ile karşılaşt-rıldığında anlamlı olarak daha düşük saptandı (\%53.5'e 
Tablo 1. iBH ve kontrol grubunun demografik verileri

\begin{tabular}{|lccc|} 
& iBH & Kontrol & P değeri \\
\hline Yaş & $41.19 \pm 13.09$ & $40.27 \pm 12.28$ & 0.637 \\
\hline Cinsiyet (Kadın) & $\% 51.3$ & $\% 56.7$ & 0.474 \\
\hline HP & $\% 52.5$ & $\% 73.3$ & 0.005 \\
\hline AG & $\% 3.1$ & $\% 15$ & 0.001 \\
\hline IM & $\% 1.9$ & $\% 11.7$ & 0.002 \\
\hline
\end{tabular}

IBH: Inflamatuvar bağırsak hastalığı, HP: Helicobacter pylori, AG: Atrofik gastrit, IM: Intestinal metaplazi.

karşı \%73.3 ve $p=0.014)$. ÜK grubunda AG oranı kontrol grubu ile karşılaştırılığında anlamlı olarak daha düşük saptandı (\%3.5'e karşı \%15 ve $p=0.013$ ). ÜK grubunda iM oranı kontrol grubu ile karşılaştıııldığında anlamlı olarak daha düşük saptandı (\%2.3'e karşı \%11.7 ve $p=$ 0.021) (Tablo 2).

$\mathrm{CH}$ grubunda $H$. pylori oranı kontrol grubu ile karşılaştırıldığında anlamlı olarak daha düşük saptandı (\%51.4'e karşı \%73.3 ve $p=0.009)$. CH grubunda AG oranı kontrol grubu ile karşılaştıııldığında anlamlı olarak daha düşük saptandı (\%2.7'ye karşı \%15 ve $p=0.008)$. CH grubunda IM oranı kontrol grubu ile karşılaştııılı̆̆ında anlamlı olarak daha düşük saptandı (\%1.4'e karşı \%11.7 ve $p=$ 0.009) (Tablo 3).

Tablo 2. Ülseratif kolit ve kontrol grubunun demografik verileri

\begin{tabular}{|cccc|}
\hline & ÜK & Kontrol & P değeri \\
\hline HP & $\% 53.5$ & $\% 73.3$ & 0.014 \\
\hline AG & $\% 3.5$ & $\% 15$ & 0.013 \\
\hline IM & $\% 2.3$ & $\% 11.7$ & 0.021 \\
\hline
\end{tabular}

ÜK: Ülseratif kolit, HP: Helicobacter pylori, AG: Atrofik gastrit, IM: Intestinal metaplazi.

Tablo 3. Crohn hastalığı ve kontrol grubunun demografik verileri

\begin{tabular}{|cccc|}
\hline & CH & Kontrol & P değeri \\
\hline HP & $\% 51.4$ & $\% 73.3$ & 0.009 \\
\hline AG & $\% 2.7$ & $\% 15$ & 0.008 \\
\hline IM & $\% 1.4$ & $\% 11.7$ & 0.009 \\
\hline
\end{tabular}

CH: Crohn hastalığı, HP: Helicobacter pylori, AG: Atrofik gastrit, IM: Intestinal metaplazi

\section{TARTIŞMA}

H. pylori prevalansı coğrafik bölge, yaş, ırk, etnik grup ve sosyoekonomik duruma göre \%13.4 ile \%92.6 arasında değişmektedir. Ülkemizde ise üre nefes testi ile yapılan prevalans çalışmasında $H$. pylori pozitifliği \%82.5 olarak saptanmıştır (13). Ülkemizde ülseratif kolit tanılı hastalarda HP prevalansı ise \%57.1 olarak saptanmıştır (14). Çalışmamızdaki popülasyonda IBH dışı olgularda dispepsi nedeni ile yapılan üst GIS endoskopi biyopsilerinin incelenmesinde $H$. pylori pozitifliği \%73.3 olarak saptandı. iBH grubunda $H$. pylori oranı kontrol grubu ile karşılaştırıldığında anlamlı olarak daha düşük saptandı (\%52.5'e karşı $\% 73.3$ ve $p=0.005)$. ÜK grubunda $H$. pylori oranı kontrol grubu ile karşılaştıııldığında anlamlı olarak daha düşük saptandı (\%53.5'e karşı \%73.3 ve $\mathrm{p}=0.014$ ). $\mathrm{CH}$ grubunda $H$. pylori oranı kontrol grubu ile karşlaş̧ıııldığında anlamlı olarak daha düşük saptandı (\%51.4'e karşı $\% 73.3$ ve $p=0.009$ ).

H. pylori ve IBH arasındaki ilişki tartışmalıdır. Literatür çaIışmaları incelendiğinde $H$. pylori ile IBH arasında negatif korelasyon gözlenmektedir. Bu negatif ilişkinin nedeni henüz tam olarak aydınlatılamamıştır. Bir hipotez, IBH tedavisi için kullanılan 5-ASA ve antibiyotikler gibi ilaçların H. pylori'yi ortadan kaldırabileceğini ya da baskılayabileceğini öne sürmektedir. Aynı çizgideki bir başka öneride, iBH'daki mukozal değişikliklerinin $H$. pylori kolonizasyonunu önleyebileceğidir. Alternatif bir argüman ise, H. pylori enfeksiyonun IBH oluşumu için koruyucu bir etki gösterdiğini önermektedir (12,15-20). Kayali S ve ark. 22 ayrı çalışmayı irdelediği meta-analiz sonucunda, farklı coğrafyalarda ele alınan IBH türünden bağımsız olarak, H. pylori enfeksiyonu ile IBH prevalansı arasında çarpıcı bir negatif korelasyon olduğu belirtilmiştir (21). Wu XW ve ark. Asya popülasyonunda yapılan çalışmaların meta-analizinde de benzer sonuçlar saptamışlardır. Bu meta-analizde de IBH'da daha düşük oranda $H$. pylori pozitifliği gözlenmiş ve $H$. pylori varlığının İBH için koruyucu etkisi olduğuna işaret edilmiştir (22). Lin KD ve ark. H. pylori eradikasyon tedavisinin, IBH dahil otoimmün hastalık riskinde önemli bir artış ile ilişkili olduğunu rapor etmişlerdir (23). Crohn hastalığı ve ülseratif koliti içeren IBH'nın, genetik yatkınlığı olan bireylerde konağın komensal bakterilere karşı bağışıklık tepkisinin düzensizliğinden kaynaklandığı 
bilinmektedir. Sağlıklı kontrollere kıyasla İH hastalarının gaita mikrobiyom bileşimindeki biyoçeşitlilik ve diğer spesifik dengesizliklerin azalması bu hipotezi desteklemektedir. Bu bağlamda, H. pylori enfeksiyonu ile IBH prevalansı arasında ters bir korelasyon doğrulanmıştır (24).

IBH'da H. pylori'nin düşük sıklıkta olması; esasında bu hastaların zaman zaman antibiyotik kullanımı ve daha fazla oranda proton pompa inhibitörü (PPI) kullanımı (yalancı negatiflik) ile ilgili olabilir. IBH hastalarının almış oldukları tedavilere bağlı olarak dispeptik yakınmalarının daha sık olması ve buna bağlı olarak daha fazla sıklıkla PPI kullanımı, özellikle üre nefes testi gibi $H$. pylori tarama tetkiklerinde yalancı $H$. pylori negatifliğine yol açabilmesine karşın bizim çalışmamızda direkt olarak immuno-histokimyasal yöntemler ile $H$. pylori varlığına bakılmıştır. Bu nedenle yalancı negatiflik olmadığı kanaatindeyiz. İBH tedavisinde daha önce kullanılan antibiyotikler ile düşük H. pylori sıklığını ilişkilendiren yayınlar olduğu gibi zıt yönde rapor eden makalelerde mevcuttur (25). Hem ÜK'da hem de $\mathrm{CH}^{\prime}$ da olgular tanı almadan önce diyare nedeni ile ampirik antibiyotik tedavileri alabilmektedir. Ayrıca tanı sonrasında da olgular başlangıç idame IBH tedavisi sırasında proflaktik olarak ya da kültürlerde saptanan diyare etkenlerine göre (amip, Shigella, Escherichia coli vb) terapötik olarak antibiyotik tedavisi kullanabilmektedir $(26,27)$. Genellikle rutin pratikte metranidazol, nidazol, siprofloksasin gibi antibiyotik tedavileri tercih edilmektedir ki bu tedaviler $H$. pylori eradikasyon tedavi rejimleri içerisinde de geçmektedir (26-28). Bu tedavilere bağlı olarak $H$. pylori prevalansının kontrol grubuna göre görece daha düşük olduğunu düşünmekteyiz.

\section{KAYNAKLAR}

1. Bravo D, Hoare A, Soto C, Valenzuela MA, Quest AF. Helicobacter pylori in human health and disease: Mechanisms for local gastric and systemic effects. World J Gastroenterol 2018;24:3071-89.

2. Siegel R, Ward E, Brawley O, Jemal A. Cancer statistics, 2011: the impact of eliminating socioeconomic and racial disparities on premature cancer deaths. CA Cancer J Clin 2011;61:212-36.

3. Watari J, Chen N, Amenta PS, et al. Helicobacter pylori associated chronic gastritis, clinical syndromes, precancerous lesions, and pathogenesis of gastric cancer development. World J Gastroenterol 2014;20:5461-73.

4. Correa P. Human gastric carcinogenesis: a multistep and multifactorial process-First American Cancer Society Award Lecture on Cancer Epidemiology and Prevention. Cancer Res 1992;52:673540.

5. Schistosomes, liver flukes and Helicobacter pylori. IARC Working Group on the Evaluation of Carcinogenic Risks to Humans. Lyon, 7-14 June 1994. IARC Monogr Eval Carcinog Risks Hum 1994;61:1241.

6. Weck MN, Brenner $\mathrm{H}$. Association of Helicobacter pylori infection with chronic atrophic gastritis: Meta-analyses according to type of disease definition. Int J Cancer 2008;123:874-81.
H. pylori enfeksiyonu, kronik AG ve İM ile güçlü bir ilişki gösterir. H. pylori mide mukozasında kolonizasyonu sonrasında birçok mekanizma ile konak defansı yenerek kronik mukozal enflamasyona neden olmaktadır. Kronik mukozal $H$. pylori enfeksiyonu lokal immün reaksiyon sonucu sırası ile zaman içinde kronik gastrit, atrofik gastrit ve intestinal metaplaziye yol açmaktadır. Kronik gastrit döneminde $H$. pylori eradikasyonu sayesinde atrofik gastrit ve intestinal metaplazi gelişimi ise önlenebilmektedir $(1,6,28,29)$. Çalışmamızda İBH grubunda, kontrol grubuna göre daha az sıklıkla AG ve IM gibi mide mukozasındaki histolojik değişiklikler izlenmiştir. Bu durum IBH tanılı olguların medikal tedavileri sırasında almakta olduğu antibiyotik tedavileri sayesinde gastrik mukozalarındaki kolonize $H$. pylori'lerin eradikasyonu ile açıklanabilir.

Sonuç olarak; çalışmamızda İH grubunda anlamlı olarak daha düşük oranda $H$. pylori pozitifliği saptanmıştır. İBH grubunda daha düşük oranda AG ve IM varlığı saptanmıştır. İBH'da bu düşük $H$. pylori oranının olası nedenleri İBH'nın immünopatolojik özellikleri, İBH'da kullanılan 5-aminosalisilik asit (5-ASA) ve antibiyotikler gibi ilaçlar olabilir. İBH hastalarında tedavide kullanılan antibiyotikler ve 5 ASA'nın $H$. pylori sıklığını azaltarak daha az sıklıkla $A G$ ve İM gelişimine yol açtığını düşünmekteyiz.

Etik Kurul: Bu çalışma Erzurum Bölge Eğitim ve Araştırma Hastanesi Klinik ve Araştırmalar Etik Kurulunun 2019/16-148 karar no'lu onayı sonrasında gerçekleştirilmiştir.

Çıkar Çatışması: Tüm yazarlar herhangi bir çıkar çatışması olmadığını kabul ederler.

7. Loftus EV Jr. Clinical epidemiology of inflammatory bowel disease: incidence, prevalence, and environmental influences. Gastroenterology 2004;126:1504-17.

8. Quetglas EG, Mujagic Z, Wigge $S$, et al. Update on pathogenesis and predictors of response of therapeutic strategies used in inflammatory bowel disease. World J Gastroenterol 2015;21:12519-43.

9. El-Omar E, Penman I, Cruikshank G, et al. Low prevalence of Helicobacter pylori in inflammatory bowel disease: association with sulphasalazine. Gut 1994;35:1385-8.

10. Pearce CB, Duncan HD, Timmis L, Green JR. Assessment of the prevalence of infection with Helicobacter pylori in patients with inflammatory bowel disease. Eur J Gastroenterol Hepatol 2000;12:43943.

11. Piodi LP, Bardella M, Rocchia C, et al. Possible protective effect of 5-aminosalicylic acid on Helicobacter pylori infection in patients with inflammatory bowel disease. J Clin Gastroenterol 2003;36:225 .

12. Triantafillidis JK, Gikas A, Apostolidiss N, et al. The low prevalence of Helicobacter infection in patients with inflammatory bowel disease could be attributed to previous antibiotic treatment. Am J Gastroenterol 2003;98:1213-4 
13. Ozaydin N, Turkyilmaz SA, Cali S. Prevalence and risk factors of Helicobacter pylori in Turkey: a nationally-representative, cross-sectional, screening with the ${ }^{13} \mathrm{C}-$ Urea breath test. BMC Public Health 2013;13:1215.

14. Caner $S$, Altinbaş A, Yeşil $Y$, et al. The relation between Helicobacter pylori and ulcerative colitis. Turk J Med Sci 2014;44:820-3.

15. D'Incà R, Sturniolo $G$, Cassaro $M$, et al. Prevalence of upper gastrointestinal lesions and Helicobacter pylori infection in Crohn's disease. Dig Dis Sci 1998;43:988-92.

16. Parente F, Molteni P, Bollani S, et al. Prevalence of Helicobacter pylori infection and related upper gastrointestinal lesions in patients with inflammatory bowel diseases. A cross-sectional study with matching. Scand J Gastroenterol 1997;32:1140-6.

17. Castaño-Rodríguez N, Kaakoush NO, Lee WS, Mitchell HM. Dual role of Helicobacter and Campylobacter species in IBD: a systematic review and meta-analysis. Gut 2015 Oct 27. pii: gutjnl-2015-310545.

18. Roka K, Roma E, Stefanaki K, et al. The value of focally enhanced gastritis in the diagnosis of pediatric inflammatory bowel diseases. J Crohns Colitis 2013;7:797-802.

19. Sonnenberg A, Melton SD, Genta RM. Frequent occurrence of gastritis and duodenitis in patients with inflammatory bowel disease. Inflamm Bowel Dis 2011;17:39-44.

20. Halme $L$, Kärkkäinen $P$, Rautelin $H$, Kosunen TU, Sipponen $P$. High frequency of helicobacter negative gastritis in patients with Crohn's disease. Gut 1996;38:379-83.

21. Kayali S, Gaiani F, Manfredi M, et al. Inverse association between Helicobacter pylori and inflammatory bowel disease: myth or fact? Acta Biomed 2018;89(9-S):81-6.
22. Wu XW, Ji HZ, Yang MF, Wu L, Wang FY. Helicobacter pylori infection and inflammatory bowel disease in Asians: a meta-analysis. World J Gastroenterol 2015;21:4750-6.

23. Lin KD, Chiu GF, Waljee AK, et al. Effects of anti-Helicobacter pylori therapy on incidence of autoimmune diseases, including inflammatory bowel diseases. Clin Gastroenterol Hepatol 2019;17:1991-9.

24. Pellicano R, laniro G, Fagoonee S, Settanni CR, Gasbarrini A. Review: Extragastric diseases and Helicobacter pylori. Helicobacter 2020;25(Suppl 1):e12741.

25. Prónai L, Schandl L, Orosz Z, Magyar P, Tulassay Z. Lower prevalence of Helicobacter pylori infection in patients with inflammatory bowel disease but not with chronic obstructive pulmonary disease - antibiotic use in the history does not play a significant role. Helicobacter 2004;9:278-83.

26. Harbord M, Eliakim R, Bettenworth D, at al; European Crohn's and Colitis Organisation [ECCO]. Third European Evidence-based Consensus on Diagnosis and Management of Ulcerative Colitis. Part 2: Current Management. J Crohns Colitis 2017;11:769-84.

27. Gomollón F, Dignass A, Annese V, et al; ECCO. 3rd European Evidence-based Consensus on the Diagnosis and Management of Crohn's Disease 2016: Part 1: Diagnosis and Medical Management. J Crohns Colitis 2017;11:3-25.

28. Malfertheiner P, Megraud F, O'Morain CA, et al; European Helicobacter and Microbiota Study Group and Consensus panel. Management of Helicobacter pylori infection-the Maastricht V/Florence Consensus Report. Gut 2017;66:6-30.

29. Kim N. Chemoprevention of gastric cancer by Helicobacter pylori eradication and its underlying mechanism. J Gastroenterol Hepatol 2019;34:1287-95. 